\title{
One-Dimensional Integer Cutting Stock Problems
}

\author{
Gerhard Wäscher, Technische Universität Braunschweig
}

Papers on cutting stock problems belong to the earliest references (Kantorovich 1939) in the field of Operations Research. Today, there is still a considerable interest in the field, which can be seen from the constantly growing number of publications.

One area which has been focused by several researchers during the last few years was the questions of how to generate integer solutions to the standard one-dimensional cutting stock problem (SCSP). Research on this subject may have been attracted by the apparent contradiction that on one hand the generation of an optimum integer solution has proven to be difficult (Gilmore 1979) while on the other hand the integer round-up property seems to hold for almost any real-world problem (Marcotte 1985).

In this paper the state-of-the-art in solving the SCSP is reported. Both exact solution methods (Branch-and-Bound) and heuristic procedures (round-off methods, repeated pattern exhaustion techniques, bin packing algorithms) are presented and compared with respect to their performance in solving a series of problems, taken from real world applications and from literature.

Our test results indicate that small and medium-sized problems can be solved to an optimum by means of standard ILP-codes when specifying simple lower bound on the objective function value. For large problems round-off methods often generate optimum solutions, while other heuristic methods only provide non-competitive solutions far from optimum.

Finally, open questions and directions for future research are pointed out.

\section{Literature}

Gilmore, P.C. (1979):

Cutting Stock, Linear Programming, Knapsacking, Dynamic Programming and Integer Programming Some Interactions. Annals of Discrete Mathematics 4, pp. 217 - 235.

Kantorovich, L.V. (1939):

Mathematical Methods of Organizing and Planning Production. Management Science 6, 1960, pp. 366422. (Translation of a report given on May 13, 1939, at the Leningrad State University). 